ACCEPTED MANUSCRIPT

\title{
Freeform 3D printing using a continuous viscoelastic supporting matrix
}

To cite this article before publication: Sónia Gonçalves Patrício et al 2020 Biofabrication in press https://doi.org/10.1088/1758-5090/ab8bc3

\section{Manuscript version: Accepted Manuscript}

Accepted Manuscript is "the version of the article accepted for publication including all changes made as a result of the peer review process, and which may also include the addition to the article by IOP Publishing of a header, an article ID, a cover sheet and/or an 'Accepted Manuscript' watermark, but excluding any other editing, typesetting or other changes made by IOP Publishing and/or its licensors"

This Accepted Manuscript is @ 2020 IOP Publishing Ltd.

During the embargo period (the 12 month period from the publication of the Version of Record of this article), the Accepted Manuscript is fully protected by copyright and cannot be reused or reposted elsewhere.

As the Version of Record of this article is going to be / has been published on a subscription basis, this Accepted Manuscript is available for reuse under a CC BY-NC-ND 3.0 licence after the 12 month embargo period.

After the embargo period, everyone is permitted to use copy and redistribute this article for non-commercial purposes only, provided that they adhere to all the terms of the licence https://creativecommons.org/licences/by-nc-nd/3.0

Although reasonable endeavours have been taken to obtain all necessary permissions from third parties to include their copyrighted content within this article, their full citation and copyright line may not be present in this Accepted Manuscript version. Before using any content from this article, please refer to the Version of Record on IOPscience once published for full citation and copyright details, as permissions will likely be required. All third party content is fully copyright protected, unless specifically stated otherwise in the figure caption in the Version of Record.

View the article online for updates and enhancements. 


\title{
Freeform 3D Printing using a Continuous Viscoelastic Supporting Matrix
}

\author{
Sónia G. Patrício ${ }^{1,4,}{ }^{*}$, Liliana R. Sousa ${ }^{1,2,4}$, Tiago R. Correia ${ }^{1}$, Vítor M. Gaspar ${ }^{1}$, Liliana \\ S. Pires ${ }^{2,3}$, Jorge L. Luís ${ }^{2,3}$, José M. Oliveira ${ }^{2,3}$ and João F. Mano ${ }^{1, *}$ \\ ${ }^{1}$ Department of Chemistry, CICECO - Aveiro Institute of Materials, University of Aveiro, Campus \\ Universitário de Santiago, 3810-193 Aveiro, Portugal \\ ${ }^{2}$ ESAN - School of Design, Management and Production Technologies Northern Aveiro, Estrada do \\ Cercal 449, 3720-509 Santiago de Riba-Ul, Portugal \\ ${ }^{3}$ Department of Materials \& Ceramic Engineeering, CICECO - Aveiro Institute of Materials, University \\ of Aveiro, Campus Universitário de Santiago, 3810-193 Aveiro, Portugal \\ ${ }^{4}$ These authors contributed equally to this work. \\ * Authors to whom any correspondence should be addressed. \\ E-mail: sgpatricio@ua.pt and jmano@ua.pt
}

Received xxxxxx

Accepted for publication $\mathrm{xxxxxx}$

Published xxxxxx

\begin{abstract}
Embedded bio-printing has fostered significant advances toward the fabrication of soft complex tissue-like constructs, by providing a physical support that allows the freeform shape maintenance within the prescribed spatial arrangement, even under gravity force. Current supporting materials still present major drawbacks for up-scaling embedded 3D bio-printing technology towards tissue-like constructs with clinically relevant dimensions. Herein, we report a a cost-effective and widely available supporting material for embedded bio-printing consisting on a continuous pseudo-plastic matrix of xanthan-gum (XG). This natural polisaccharide exhibits peculiar rheological properties that have enabled the rapid generation of complex volumetric 3D constructs with out of plane features. The freedom of design within the three orthogonal axes through the independent and controlled bio-printing process opens new opportunities to produce on demand large arbitrary shapes for personalized medicine. Additionally, we have demonstrated the versatile functionality of XG as a photocurable gel reservoir to engineer perfused cell-laden hydrogel constructs, addressing other practical biomedical applications such as in vitro models and organ-on-chip platforms.
\end{abstract}

Keywords: Embedded 3D printing, supporting materials, continuous matrix, freeform structures, hydrogels

\section{Introduction}

In the current era of 3D bio-printing, cell-laden hydrogels are commonly employed as bioinks to generate spatially organized cell-material constructs that can be used in bottomup tissue engineering to recapitulate the architectural features of native tissues [1-3]. However, cell-laden hydrogels are soft hybrid materials with limited mechanical strength to maintain the prescribed 3D shape integrity and fidelity under gravity force [4]. Conventional approaches to improve the printability of hydrogel-based bioinks resort to the modulation of their rheological properties and crosslinking densities, which could have detrimental consequences for cell viability, proliferation and differentiation [5, 6]. To overcome this limitation, an emerging strategy is the use of supporting materials to provide a physical support during the $3 \mathrm{D}$ printing process, ensuring shape maintenance of the freeform printed structures with the 
desired spatial control [7]. Such embedded extrusion-based printing approach has already enabled the fabrication of hierarchical hollow structures and complex 3D constructs with large overhangs and internal voids that have still remained mechanically robust after being removed from the supporting matrix [8-11]. Current sacrificial supporting materials consist of microparticle slurries $[9,10,12,13]$ and jammed microgels, namely carbopol [8, 14, 15] and gelatin microparticles developed by coacervation approach [11]. Despite the opportunities provided by the microparticle-based supporting materials, carbopol is not compatible with some hydrogel crosslinking agents [16], while microparticle slurry production encompasses labor-intensive and time-consuming processes with yield losses (e.g. mechanical blending, centrifugation, vacuum filtration) $[9,11,13]$, hindering its scale-up to generate tissue-like constructs with clinically relevant dimensions. Furthermore, strategies to recover 3D printed structures from supporting materials has proven challenging for further clinical translation, requiring delicate removal processes as temperature $[9,11]$, enzymatic or chemical degradation $[10,12]$ that could compromise cell viability. Alternatively, host-guest hydrogel supporting materials are continuous matrices that enable the fast and safe removal of 3D printed structures due to the noncovalent and reversible bonds of the supramolecular assembly hydrogel [17, 18]. However, a major drawback is still the printing speed towards manufacturing scalability (from 10 to $20 \mathrm{~mm} \cdot \mathrm{min}^{-1}$ ). Also, host-guest hydrogels require several chemical modification steps to form noncovalent and reversible bonds, which is challenging for large scale production. Therefore, current features of supporting materials for extrusion-based bioprinting are still too limited for the generation of fast, large and well-defined tissue-like constructs, thereby constraining the scaling up towards clinical translation.

In order to circumvent the recurrent challenges in embedded bio-printing via extrusion-based approach, we herein report a widely available and cost-effective natural polymer based on highly pseudo-plastic solutions of xanthan gum (XG). Shear-thinning pseudo-plasticity results from the order-disorder conformational transition of XG molecules [19]. The high molecular weight of this natural polysaccharide is responsible for the highly ordered polymer network, stabilized through hydrogen bonds and polymer entanglement, which provide high solution viscosity at low shear rates. Under a shear force, intermolecular bonds are progressively disrupted resulting in a lower viscosity solution, which recovers its initial viscosity when the applied shear is removed. The pseudo-plasticity behavior supplies outstanding suspending properties [20], thus making XG widely employed as an efficient stabilizer in food, cosmetic and pharmaceutical industries [21]. We therefore hypothesize that such conformational transition at different shear rates can leverage xanthan gum as a simple supporting material for engineering freeform suspended 3D bio-printed structures. Xanthan-gum was recently employed in embedded bio-printing as a dispersant agent to suspend and stabilize the granular supporting matrix [10]. However, XG rheological properties as standalone supporting material are still disregarded. Hence, in a proof-of-experiment, we demonstrated the versatile functionality of $\mathrm{XG}$ as supporting material by two distinct embedded bio-printing approaches (figure 1): either as sacrificial viscous supporting matrix to generate highly complex out-of-plane volumetric structures (figure 1(A)), or as a permanent supporting material being part of the final

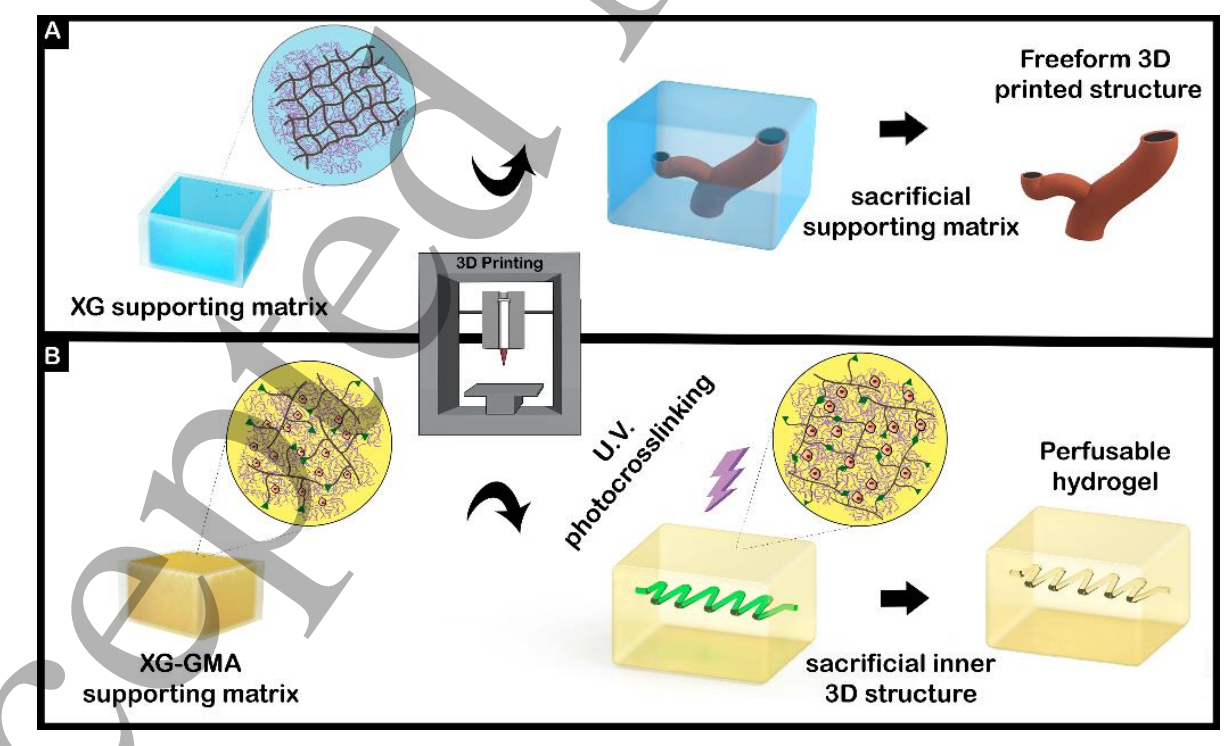

Figure 1. Schematics of the proposed embedded (bio)printing approaches for XG-based matrices: (A) as a sacrificial matrix providing physical support for the printed freeform structure and (B) as a permanent matrix providing support for a temporary 3D structure, which is then sacrificed within the cured supporting matrix, being part of the final 3D construct. 
construct (figure (1B)). In both approaches, alginate was selected as hydrogel ink due to its fast and reversible ionic gelation by divalent cations, biocompatibility and low cost $[22,23]$.

\section{Methods}

\subsection{Cell culture}

L929 mouse fibroblasts (ATCC ® CRL-6364TM) were seeded in $125 \mathrm{~cm}^{2}$ t-flasks using Dulbecco's Modified Eagle's Medium Low Glucose (DMEM-LG, ThermoFisher Scientific), supplemented with $10 \%$ (v/v) fetal bovine serum (FBS, ThermoFisher Scientific) and (1\% (v/v) antibiotic/antimycotic (ThermoFisher Scientific) in controlled atmosphere of $5 \% \mathrm{CO}_{2}$ and $37{ }^{\circ} \mathrm{C}$. Upon attaining confluence, cells were trypsinized and resuspended in alginate ink or XGGMA supporting matrix.

\section{2 (Bio)ink formulations}

An aqueous solution of $3.5 \%(\mathrm{w} / \mathrm{v})$ sodium alginate (BioChemica, MM 10000-600000 $\mathrm{g} \mathrm{mol}^{-1}$ ) with $0.1 \%$ (w/v) of food dye was prepared to print complex freeform structures. To evaluate XG biocompatibility, L929 mouse fibroblasts $\left(5 \times 10^{6}\right.$ cell $\left.\mathrm{mL}^{-1}\right)$ were added in $2.0 \%(\mathrm{w} / \mathrm{v})$ alginate, previously dissolved in complete cell culture medium.

\subsection{Preparation of XG supporting matrix}

Firstly, $50 \mathrm{mM} \mathrm{CaCl} 2$ (BioChemica) was dissolved in distilled water. Afterwards, $1.5 \%(w / v)$ of XG (Doves Farm Foods Ltd, distributed by Bricer Unipessoal, Portugal) was slowly added to avoid the formation of lumps and kept under vigorous stirring overnight, at room temperature. For 3D bioprinting of cell laden filaments, $\mathrm{CaCl}_{2}$ was dissolved in supplemented culture medium.

\subsection{Rheological characterization}

Rheological measurements of XG supporting matrix were performed on a Kinexus Lab+ rheometer (Malvern Panalytical), by using a $20 \mathrm{~mm}$ diameter parallel plate geometry and $0.5 \mathrm{~mm}$ gap at $25^{\circ} \mathrm{C}$. The variation of viscosity was measured with a continuously ramped shear rate $(0.1$ to $100 \mathrm{~s}^{-1}$ ) to study shear-thinning properties. To determine the linear viscoelastic region (LVR), strain amplitude sweep measurements $(0.1$ to $1000 \%)$ were performed at a frequency of $1 \mathrm{~Hz}$. Oscillatory frequency sweep measurements $(0.01$ to $100 \mathrm{~Hz}$ ) were then conducted at constant $3 \%$ strain amplitude to determine the storage $\left(\mathrm{G}^{\prime}\right)$ and loss $\left(\mathrm{G}^{\prime \prime}\right)$ moduli.

\subsection{D Bio-printing}

Complex freeform structures were printed using a bioplotter pneumatic dispensing system (3D-
Bioplotter ${ }^{\circledR}$ Developer Series, Envisiontec). Typically, extrusion was carried out through $25 \mathrm{G}$ needle tips with a pneumatic pressure of $30 \mathrm{kPa}$ and printing speed of $30 \mathrm{~mm} \mathrm{~s}^{-1}$. Structures were designed with SolidWorks software and then exported to STL format files. These files were processed by Perfactory RP ${ }^{\circledR}$ Software Suite and sliced into $200 \mu \mathrm{m}$ thick layers to generate G-code instruction for the bioplotter.

\subsection{Perfused cell-laden supporting hydrogel}

Methacrylated xanthan gum (XG-GMA) was synthesized as previously reported [30]. Briefly, $1.0 \mathrm{~g}$ of xanthan gum was dissolved at $0.5 \%(\mathrm{w} / \mathrm{v})$ in distilled water under vigorous stirring, at room temperature. Afterwards, $8 \mathrm{~mL}$ of glycidyl methacrylate (TCI Chemicals) was added dropwise. The reaction was carried out at $60^{\circ} \mathrm{C}$ for $12 \mathrm{~h}$. Afterwards, XGGMA was precipitated with ethanol and then dissolved in water. The solution was dialyzed (MWCO 6-8 $\mathrm{kDa}$ regenerated celulose membrane) against water for 5 days, at $\mathrm{RT}$, in the dark (changing water 3 times per day). The resulting solution was frozen at $-80{ }^{\circ} \mathrm{C}$ followed by freeze-drying (-86 ${ }^{\circ} \mathrm{C}$, Telstar Lyoquest) and stored at $4{ }^{\circ} \mathrm{C}$, until further use. The obtained XG-GMA was characterized by proton nuclear magnetic resonance $\left({ }^{1} \mathrm{H}\right.$ NMR, $\mathrm{D}_{2} \mathrm{O}$ solvent) and Fourier transformed infrared spectroscopy (FTIR-ATR). NMR spectra were recorded at $70^{\circ} \mathrm{C}$ on a Bruker Advance III 300 $\mathrm{MHz}$ spectrometer. FTIR spectra were obtained in a Brucker Tensor 27 spectrometer (256 scans, $4 \mathrm{~cm}^{-1}$ resolution).

XG-GMA supporting matrix was prepared by dissolving $0.5 \%(\mathrm{w} / \mathrm{v})$ of methacrylate $\mathrm{XG}$ in a phosphate-buffered saline solution (PBS, ThermoFischer Scientific) of $0.1 \%(\mathrm{w} / \mathrm{v}) 2$ hydroxy-4'-(2-hydroxyethoxy)-2-methylpropiophenone (Irgacure 2959, Sigma-Aldrich), supplemented with $50 \mathrm{mM}$ $\mathrm{CaCl}_{2}$. Afterwards, L929 cells were mixed with XG-GMA supporting matrix, at a final density of $20 \times 10^{6}$ cells $\mathrm{mL}^{-1}$. After printing alginate single filaments, $\mathrm{XG}-\mathrm{GMA}$ supporting matrix was photocrosslinked upon UV light exposure (OmniCure S2000, 320-500 nm filter, $60 \mathrm{~s}, 100 \mathrm{~mW} / \mathrm{cm}^{2}$ ). Then, to liquefy the alginate filament crosslinked hydrogels were incubated in culture medium supplemented with $20 \mathrm{mM}$ ethylenediaminetetraactetic acid (EDTA, Sigma-Aldrich) for 4h.

\subsection{Live/dead staining}

To evaluate cell viability, cell-laden alginate filaments and cross-sections of cell-laden XG-GMA hydrogels were incubated in Calcein-AM/Propidium iodide (PI) (Live/Dead kit, ThermoFisher Scientific) for $20 \mathrm{~min}$, according to the manufacturer's protocol. Widefield (Zeiss, Axio imager 2) and confocal laser scanning (Zeiss, LSM 880 Airy Scan) microscope systems were used to image the stained cell-laden structures. Acquired data was processed in Zeiss ZEN v2.3 blue edition software. 


\section{Results and Discussion}

\subsection{Continuous XG matrix for freeform 3D structures}

To illustrate the ability of XG to hold non-self-supporting structures, alginate constructs were $3 \mathrm{D}$ printed in a gravitydefying spatial arrangement into $1.5 \%(\mathrm{w} / \mathrm{v}) \mathrm{XG}$ support matrix, supplemented with $\mathrm{CaCl}_{2}(50 \mathrm{mM})$ for alginate ionic crosslinking and a green dye for better visualization. Firstly, we created a macroscale 3D asymmetric object in which the bottom of the structure $(\sim 1 \mathrm{~cm})$ was largely narrower than the top (>5 cm) (figure $2 \mathrm{~A}$ (i)-(iii)), where the line of gravity falls outside the structure support base. Notably, during printing process, XG support material fluidizes locally owing to the shear force induced by the nozzle motion (Movie S1, supporting information), allowing the alginate ink deposition. When the printing nozzle moves away from the local extrusion, the shear force is locally removed and the extruded alginate is upheld within XG matrix, which recovers its initial viscosity by reestablishing intermolecular hydrogen bonding networks. Such noncovalent interactions are an intrinsic properties of XG polysaccharide, one of the greatest advantages over host-guest hydrogel supporting materials that requires chemical modifications for further supramolecular assembly.

At the end of the 3D printing process, the 3D asymmetric hydrogel structure doesn't collapse by the gravity effect, highlighting the outstanding suspending properties of $\mathrm{XG}$ supporting matrix. Likewise, the continuous XG fluid matrix has also allowed the generation of a volumetric truncated tube with free-floating hollow vessels (figure $2 \mathrm{~B}$ (i)-(iii)). Interestingly, despite the 3D artery-like tree being consecutively built on 2D patterned layers in a layer-by-layer fashion, the XG supporting matrix still enable the fabrication of out-of-plane branches, allowing to print structures beyond the commonly 2.5D. Moreover, this artery-like tree with out of plane branches was printed into the XG supporting matrix with significantly improved speed $\left(30 \mathrm{~mm} \cdot \mathrm{s}^{-1}\right.$ for a centimeter scale construct), without compromising the printing resolution of the complex 3D constructs. This result is particularly attractive when compared with those obtained for the already reported supporting materials to be used for extrusion-based approach, ranging from $0.17-0.33$ (spiral structures) $[17,18]$ to $3-10 \mathrm{~mm} \cdot \mathrm{s}^{-1}$ (ventricle) [11] for supramolecular hydrogels and jammed gelatin microparticles, respectively. Therefore, XG matrix offers the unique possibility to create volumetric out-of-plane constructs with high speed and printing fidelity, paving the way for up-scaling construct dimensions to clinically relevant sizes, which is still an unmet challenge in the extrusion-based bio-printing field.

In a first attempt, these complex centimeter-scale constructs were printed with alginate ink, where crosslinking occurs within XG supporting matrix along with the extrusion process due to the compatibility of XG supporting matrix with
$\mathrm{CaCl}_{2}$ salt (Movie $\mathrm{S} 1$, supporting information). According to the stability of XG solutions over broad salt concentrations, $\mathrm{pH}$ and presence of most enzymes [19], we envision that XG supporting matrix may act as an universal reservoir for several crosslinking agents, thus enabling 3D printing multiple materials that virtually undergo different gelation mechanisms (e.g. ionic, pH, enzymatic and through covalent bonds).

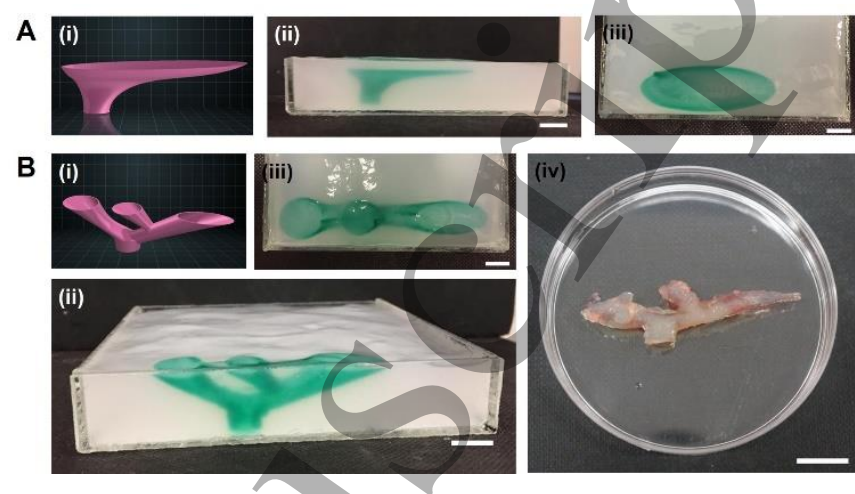

Figure 2. (A) and (B) Freeform suspended 3D structures printed in a gravity-defying spatial arrangement into $1.5 \%(\mathrm{w} / \mathrm{v}) \quad \mathrm{XG}$ supporting matrix: (i) CAD model, (ii) and (iii) front and top view, respectively; iv) Out of plane truncated tube after removing from XG supporting matrix, showing that alginate 3D printed layers are well fused together and mechanically stable. Scale bars $1 \mathrm{~cm}$.

Importantly, in embedded 3D bio-printing, the final printed constructs must be removed from the supporting matrix without jeopardizing its structural integrity or even the viability of encapsulated cells. Herein, the truncated tube with out of plane free-floating hollow vessels was easily removed from XG support matrix by immersing the container in water. The rapid and efficient dilution of XG solution released the 3D printed construct without damages, preserving the hollow parts (Figure 2 B (iv), Movie S2 supporting information). Furthermore, after removal, the alginate layers of the asymmetric truncated tube were well fused together, without delamination. In the presence of cells, 3D structures can be immersed in culture medium to wash the hybrid construct, maintaining cell viability. With this approach complex cellladen structures can be easily released from XG matrix in mild conditions in contrast to other current embedded bio-printing approaches, which comprise chemical and/or enzymatic degradation $[10,12]$.

The rheological properties of XG support matrix are critical during the deposition and fixation of the extrudate. The XG supporting matrix exhibited pseudo-plasticity, in which the fluid viscosity decreased exponentially with increasing shear rate (figure $3 \mathrm{~A}$ (i)). Such gel-like behavior was predominantly elastic, at a strain of 3\% (figure $3 \mathrm{~A}$ (ii) and figure S1, supporting information). However, with the strain increasing, the loss modulus $\left(G^{\prime \prime}\right)$ surpassed the elastic one $\left(G^{\prime}\right)$ at approximately $126 \%$ strain, where XG matrix underwent a 
transition from a gel-like elastic state to a liquid-like state (figure S1, supporting information). At this point, the linear viscoelastic region is vanished and $G$ ' becomes strain dependent. We therefore hypothesized this shear-yielding is similar to the shear force applied by the nozzle motion within XG supporting matrix, allowing it to fluidize with little resistance for the extrusion of the alginate ink.

To demonstrate the freedom of design provided by $\mathrm{XG}$, a continuous filament was freely drawn in any direction of the 3D space (figure 3B and Movie S3 in supporting information), remaining stable within $\mathrm{XG}$. supporting matrix, even without a bottom layer sustaining its own weight. Such demonstration highlights the unique ability of $\mathrm{XG}$ as a continuous phase to provide physical support for the construction of nonplanar 3D arbitrary shapes. This is particularly attractive for open-source $3 \mathrm{D}$ printers due to their printing head for moving freely in the $\mathrm{X}, \mathrm{Y}$, and $\mathrm{Z}$ axes.
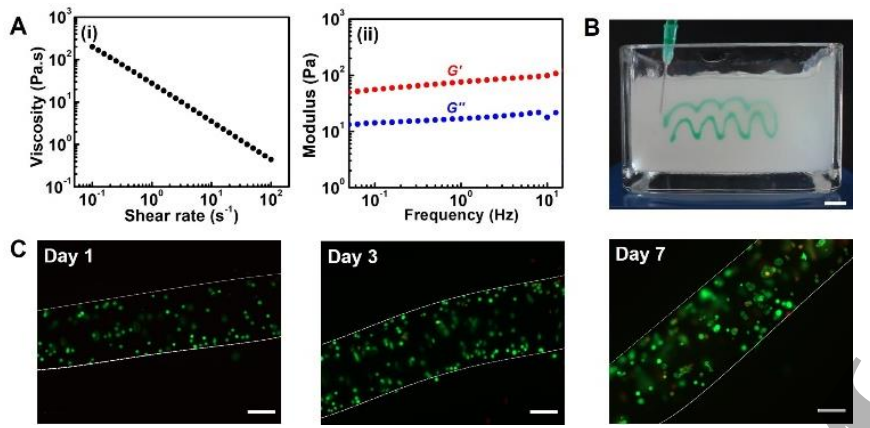

Figure 3. (A) Rheological characterization of XG supporting matrix: (i) shear-thinning behavior where viscosity decreases with increasing shear rates and (ii) frequency sweep at a strain of $3 \%$ (within the linear viscoelastic region, Figure S1); (B) Free hand design of a continuous filament, in any direction of the 3D space, within the supporting matrix, highlighting the outstanding suspending properties of XG - Scale bar $1 \mathrm{~cm}$; (C) Fluorescence micrographs of L929 cell laden alginate filaments extruded into XG supporting material, at different time points: (i) 1 , (ii) 3 and (iii) 7 days. Live cells- green channel (Calcein-AM); Dead cells red channel (PI). Scale bars $200 \mu \mathrm{m}$.

To demonstrate the cell-friendly environment of $\mathrm{XG}$, mouse fibroblasts L929 cells (density $5 \times 10^{6}$ cells $\mathrm{mL}^{-1}$ ) were encapsulated in a $2.0 \%(\mathrm{w} / \mathrm{v})$ alginate ink and $3 \mathrm{D}$ printed as cell-laden hydrogel filaments into a $1.5 \%(\mathrm{w} / \mathrm{v}) \mathrm{XG}$ culture medium sepplemented with $10 \%$ FBS. After $1 \mathrm{~h}$, these filaments were removed from $\mathrm{XG}$ supporting matrix and incubated in growth medium for 1, 3 and 7 days. Live/Dead analysis showed that L929 cells were viable until a period of 7 days in culture (Figure $3 \mathrm{C}$ ), confirming the biocompatible nature of the XG supporting material, as well as their tolerance to the shear forces exerted during the bioprinting process.

\subsection{Photocurable XG gel reservoir for perfused hydrogel constructs}

To further illustrate the versatility of XG support matrix, we also developed a photocurable $\mathrm{XG}$ gel reservoir to design thick perfusable tissue engineering constructs, thereby addressing the recurrent challenge of vascularization and perfusion of nutrients $[9,11,17,24,25]$. To achieve this, XG was firstly modified with methacrylate functional groups (XG-GMA) (figure $4 \mathrm{~A}$ and figure $\mathrm{S} 2$, supporting information), a common chemical strategy to produce photocrosslinkable hydrogels for biomedical applications [25, 26-30]. Afterwards, an alginate hydrogel filament was printed within $0.5 \%(\mathrm{w} / \mathrm{v})$ methacrylated XG matrix, which was subsequently crosslinked upon UV light exposure $(60 \mathrm{~s}, 100$ $\mathrm{mW} / \mathrm{cm}^{2}$ ), enclosing the alginate filament within XG-GMA hydrogel matrix (figure $4 \mathrm{~B}$ ). Such alginate filament was then liquified with EDTA, leaving behind an open channel that was easily perfused with a blue dye flowing through the well defined spiral microchannel (Figure $4 \mathrm{C}$ and Movie S4, supporting information).

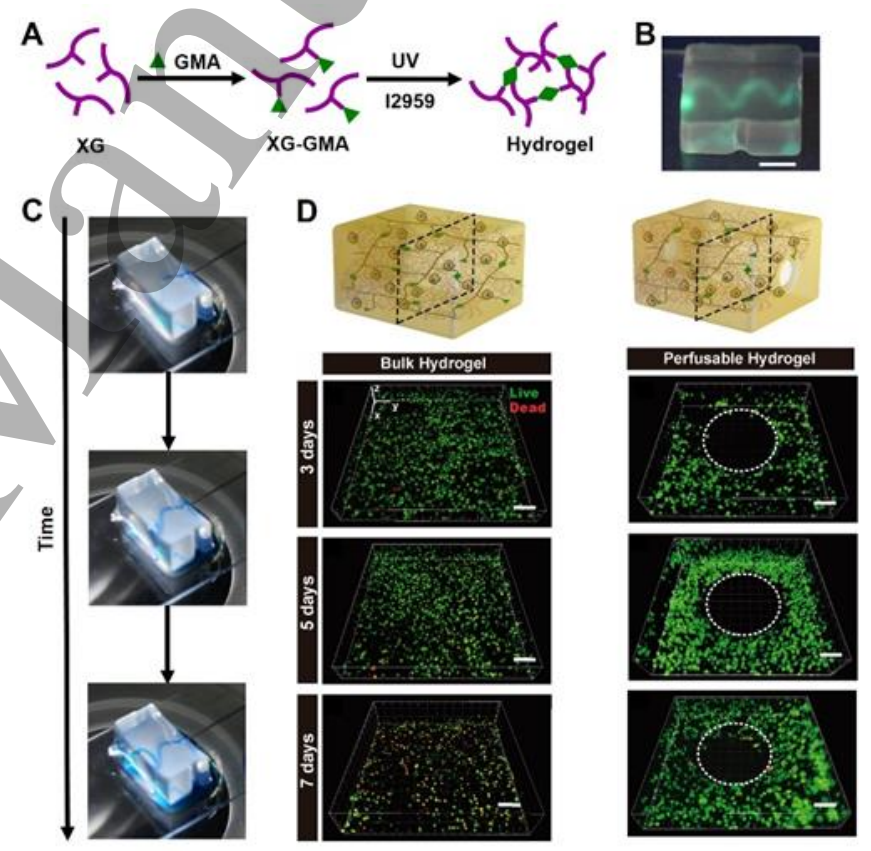

Figure 4. (A) Xanthan gum modified with methacrylate groups via epoxide ring-opening reaction of glycidyl methacrylate (GMA, green) and crosslinking of the carbon double bonds upon UV light exposure, forming a hydrogel; (B) Spiral alginate printed filament into XG-GMA supporting matrix, which covalently crosslinks around the alginate hydrogel, upon UV light irradiation; (C) time-lapse sequence of the channel perfusion (blue dye) after liquefying the alginate filament embedded within XG-GMA hydrogel; (D) Cross-section analysis of L929 cells encapsulated in $0.5 \%$ XG-GMA hydrogels: Live/Dead images for bulk (control) and perfused hydrogels. Scale bars $100 \mu \mathrm{m}$. 
To demonstrate the effectiveness of microchannels for regulating mass transport into thick hydrogel constructs, a single alginate filament $(\sim 350 \mu \mathrm{m}$, figure $\mathrm{S} 3$, supporting information) was printed into a XG-GMA supporting matrix laden with L929 cells at a density $20 \times 10^{6}$ per mL. Upon photocrosslinking and removal of the sacrificial alginate ink, perfused thick cell-laden XG-GMA hydrogels (figure S4, supporting information) were cultured in vitro up to 7 days. Cross-section analysis of perfused hydrogels (figure $4 \mathrm{D}$ ) clearly shown viable cells at different time points, with increased viability and cell clusters at 5 and 7 days, when compared to the bulk controls. Looking ahead, this embedded printing approach may be readily extended to create more complex and hierarchical vascular-mimetic networks for addressing pratical biomedical applications such as in vitro models and organ-on-a-chip platforms.

\section{Conclusions}

We proposed a new supporting material for up-scaling embedded 3D bio-printing technology that was implemented with xanthan gum, a biocompatible, wide accessible and low cost natural polysaccharide. The peculiar rheological properties of XG supporting matrix enable the arbitrary design of complex centimeter scale 3D constructs with unprecedented printing velocity, comparing to other embedded 3D printing constructs obtained via extrusionbased approach. Also, the ability of $X G$ to work as a continuous supporting matrix offers the possibility for the fabrication of volumetric 3D structures with out-of-plane features, like arteries or even other anatomic parts with intricate nonplanar architectures due to the independent and controlled 3D printing process within the the three orthogonal axes. Such 3D structures can be easily recovered from XG matrix under mild conditions without layer delamination, preserving its structural integrity.

The versatility to solubilize multiple molecules have permitted the fabrication of a photocurable XG gel reservoir to engineer perfused hydrogel constructs. We further expect that XG biocompatibility combined with the universal reservoir character for several orthogonal crosslinking mechanisms can leverage the $3 \mathrm{D}$ bio-printing of multimaterials within the same supporting matrix, as a vanguard strategy to recapitulate the heterogeneous composition of native tissues. Such approach opens unique prospects for personalized medicine.

\section{Acknowledgements}

This work was financially supported by the Programa Operacional Competitividade e Internacionalização (POCI), in the component FEDER, and by national funds (OE) through FCT/MCTES, in the scope of PANGEIA (PTDC/BTMSAL/30503/2017) and MARGEL (PTDC/BTM-
MAT/31498/2017) projects. This work was also developed within the scope of the project CICECO-Aveiro Institute of Materials (UIDB/50011/2020 \& UIDP/50011/2020) financed by national funds through the FCT/MCTES. The costs resulting from the FCT hirings is funded by national funds (OE), through FCT in the scope of the framework contract foreseen in the numbers 4,5 and 6 of the article 23 , of the Decree-Law 57/2016, of August 29, changed by Law 57/2017, of July 19. LSCM image acquisition was performed in the LiM facility of iBiMED, a node of PPBI (Portuguese Platform of BioImaging) with grant agreement number POCI-01-0145FEDER-022122.

\section{References}

[1] Clegg J R, Wagner A M, Shin S R, Hassan S, Khademhosseini A and Peppas N A 2019 Modular fabrication of intelligent material-tissue interfaces for bioinspired and biomimetic devices Prog. Mater. Sci. 106100589

[2] Moroni L, Burdick J A, Highley C, Lee S J, Morimoto Y, Takeuchi S and Yoo J J 2018 Biofabrication strategies for 3D in vitro models and regenerative medicineNat. Rev. Mater. 321

[3] Gaspar V M, Lavrador P, Borges J, Oliveira M B and Mano J F 2020 Advanced Bottom-Up Engineering of Living Architectures Adv. Mater. 321903975

[4] Williams D, Thayer P, Martinez H, Gatenholm E and Khademhossein A 2018 A perspective on the physical, mechanical and biological specifications of bioinks and the development of functional tissues in 3D bioprinting Bioprinting 919

[5] Malda J, Visser J, Melchels F P, T. Jüngst, Hennink W E, Dhert W J A, Groll J and Hutmacher D W 2013 Adv. Mater. Engineering Hydrogels for Biofabrication 255011

[6] Ouyang L, Highley C B, Sun W and Burdick J A 2017 A Generalizable Strategy for the 3D Bioprinting of Hydrogels from Nonviscous Photo-crosslinkable Inks Adv. Mater. 29 1604983

[7] O'Bryan C S, Bhattacharjee T, Niemi S R, Balachandar S, Baldwin N, Ellison S T, Taylor C R, Sawyer W G and Angelini T E 2017 Three-dimensional printing with sacrificial materials for soft matter manufacturing MRS Bull. 42571

[8] Bhattacharjee T, Zehnder S M, Rowe K G, Jain S, Nixon R M, Sawyer W G and Angelini T E 2015 Writing in the granular gel medium Sci. Adv. 1 e1500655

[9] Hinton T J, Jallerat Q, Palchesko R N, Park J H, Grodzicki M S, Shue H -J, Ramadan M H, Hudson A R and Feinberg A W 2015 Three-dimensional printing of complex biological structures by freeform reversible embedding of suspended hydrogels $S c i$. $A d v .1$ e 1500758

[10] Noor N, Shapira A, Edri R, Gal I, Wertheim L and Dvir T 2019 3D Printing of Personalized Thick and Perfusable Cardiac Patches and Hearts Adv. Sci. 61900344

[11] Lee A, Hudson A R, Shiwarski D J, Tashman J W, Hinton T J, Yerneni S, Bliley J M, Campbell P G and Feinberg A W 2019 $3 \mathrm{D}$ bioprinting of collagen to rebuild components of the human heart Science $\mathbf{3 6 5} 482$

[12] Jeon O, Lee Y B, Jeong H, Lee S J, Wells D and Alsberg E 2019 Individual cell-only bioink and photocurable supporting 
medium for 3D printing and generation of engineered tissues with complex geometries Mater. Horiz. 61625

[13] Moxon S R, Cooke M E, Cox S C, Snow M, Jeys L, Jones S W, Smith A M and Grover L M 2017 Suspended Manufacture of Biological Structures Adv. Mater. 291605594

[14] Jin Y, Compaan A, Bhattacharjee T and Huang Y 2016 Granular gel support-enabled extrusion of three-dimensional alginate and cellular structures Biofabrication 8025016

[15] O'Bryan C S, Bhattacharjee T, Marshall S L, Sawyer W G and Angelini T E 2018 Commercially available microgels for 3D bioprinting Bioprinting 11 e00037

[16] Compaan A M, Song K and Huang Y 2019 Gellan Fluid Gel as a Versatile Support Bath Material for Fluid Extrusion Bioprinting ACS Appl. Mater. Interfaces 115714

[17] Highley C B, Song K H, Daly A C and Burdick J A 2019 Jammed Microgel Inks for 3D Printing Applications Adv. Sci. 6 1801076

[18] Song K H, Highley C B, Rouff A and Burdick J A 2018 Complex 3D-Printed Microchannels within Cell-Degradable Hydrogels Adv. Funct. Mater. 281801331

[19] Kumara A, Rao K M and Han S S 2018 Application of xanthan gum as polysaccharide in tissue engineering: A review Carbohydr. Polym. 180128

[20] C.P. Kelco, Keltrol/Kelzan, Xanthan gum book 8th edition 2007

[21] Palaniraj A and Jayaraman V 2011 Production, recovery and applications of xanthan gum by Xanthomonas campestris $J$. Food Eng. 1061

[22] Faramarzi N, Yazdi I K, Nabavinia M, Gemma A, Fanelli A, Caizzone A, Ptaszek L M, Sinha I, Khademhosseini A, Ruskin J $\mathrm{N}$ and Tamayol A 2018 Patient-Specific Bioinks for 3D Bioprinting of Tissue Engineering Scaffolds Adv. Healthcare Mater. 71701347

[23] Kirillova A, Maxson R, Stoychev G, Gomillion C T and Ionov L 2017 4D Biofabrication Using Shape-Morphing Hydrogels Adv. Mater. 291703443

[24] Grigoryan B, Paulsen S J, Corbett D C, Sazer D W, Fortin C L, Zaita A J, Greenfield P T, Calafat N J, Gounley J P, Ta A H, Johansson F, Randles A, Rosenkrantz J E, Louis-Rosenberg J D, Galie P A, Stevens K R and Miller J S 2019 Multivascular networks and functional intravascular topologies within biocompatible hydrogels Science $\mathbf{3 6 4} 458$

[25] Bertassoni L E, Cecconi M, Manoharan V, Nikkhah M, Hjortnaes J, Cristino A L, Barabaschi G, Demarchi D, Dokmeci M R, Yang Y and Khademhosseini A 2014 Hydrogel bioprinted microchannel networks for vascularization of tissue engineering constructs Lab Chip $\mathbf{1 4} 2202$

[26] Santos S C, Custódio C A and Mano J F 2018 Photopolymerizable Platelet Lysate Hydrogels for Customizable 3D Cell Culture Platforms Adv. Healthcare Mater. 71800849

[27] Bjørge I M, Costa A M S, Silva A S, Vidal J P O, Nóbrega J M and Mano J F 2018 Tuneable spheroidal hydrogel particles for cell and drug encapsulation Soft Matter 145622

[28] Klotz B J, Gawlitta D, Rosenberg A J W P, Malda J and Melchels F P W 2016 Gelatin-Methacryloyl Hydrogels: Towards Biofabrication-Based Tissue Repair Trends Biotechnol. 34394

[29] Mihaila S M, Gaharwar A K, Reis R L, Marques A P, Gomes M E and Khademhosseini A 2013 Photocrosslinkable kappa- carrageenan hydrogels for tissue engineering applications $A d v$. Healthcare Mater. 2895

[30] Huang J, Li Z, Hu Q, Chen G, Ren Y, Wu X and Ren J 2018 Bioinspired Anti-digestive Hydrogels Selected by a Simulated Gut Microfluidic Chip for Closing Gastrointestinal Fistula iScience $\mathbf{8} 40$

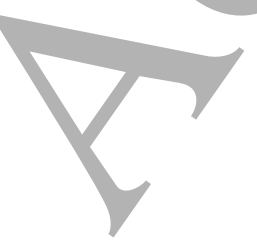

